\title{
BMJ Open Does achieving the best practice tariff improve outcomes in hip fracture patients? An observational cohort study
}

\author{
B Oakley, ${ }^{1} \mathrm{~J}$ Nightingale, ${ }^{1}$ CG Moran, ${ }^{1}$ IK Moppett ${ }^{2,3}$
}

To cite: Oakley B, Nightingale J, Moran CG, et al. Does achieving the best practice tariff improve outcomes in hip fracture patients? An observational cohort study. BMJ Open 2017;7:e014190.

doi:10.1136/bmjopen-2016014190

- Prepublication history for this paper is available online. To view these files please visit the journal online (http://dx.doi.org/10.1136/ bmjopen-2016-014190).

Received 8 September 2016 Revised 7 October 2016 Accepted 31 October 2016

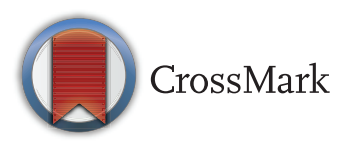

\footnotetext{
${ }^{1}$ Department of Trauma and Orthopaedics, Queens Medical Centre, Nottingham University Hospitals NHS Trust, Nottingham, UK ${ }^{2}$ Anaesthesia and Critical Care Section, Division of Clinical Neuroscience, Queen's Medical Centre, University of Nottingham, Nottingham, UK ${ }^{3}$ Department of Anaesthesia, Queen's Medical Centre, Nottingham University Hospitals NHS Trust, Nottingham, UK
}

Correspondence to B Oakley;

ben.oakley@nhs.net

\section{ABSTRACT}

Objectives: To determine if the introduction of the best practice tariff (BPT) has improved survival of the elderly hip fracture population, or if achieving BPT results in improved survival for an individual.

Setting: A single university-affiliated teaching hospital. Participants: 2541 patients aged over 60 admitted with a neck of femur fracture between 2008 and 2010 and from 2012 to 2014 were included, to create two cohorts of patients, before and after the introduction of BPT. The post-BPT cohort was divided into two groups, those who achieved the criteria and those who did not.

\section{Primary and secondary outcome measures:}

Primary outcomes of interest were differences in mortality across cohorts. Secondary analysis was performed to identify associations between individual BPT criteria and mortality.

Results: The introduction of BPT did not significantly alter overall 30-mortality in the hip fracture population (8.3\% pre-BPT vs $10.0 \%$ post-BPT; $p=0.128$ ). Neither was there a significant reduction in length of stay (15 days (IQR 9-21) pre-BPT vs 14 days (IQR 11-22); $\mathrm{p}=0.236$ ). However, the introduction of BPT was associated with a reduction in the time from admission to theatre (median 44 hours pre-BPT (IQR 24-44) vs 23 hours post-BPT (IQR 17-30); $p<0.005$ ). 30-day mortality in those who achieved BPT was significantly lower $(6.0 \%$ vs $21.0 \%$ in those who did not achieveBPT; $p<0.005)$. There was a survival benefit at 1 year for those who achieved BPT (28.6\% vs $42.0 \%$ did not achieve-BPT; $p<0.005)$. Multivariate logistic regression revealed that of the BPT criteria, AMT monitoring and expedited surgery were the only BPT criteria that significantly influenced survival.

Conclusions: The introduction of the BPT has not led to a demonstrable improvement in outcomes at organisational level, though other factors may have confounded any benefits. However, patients where BPT criteria are met appear to have improved outcomes.

\section{INTRODUCTION}

Hip fractures are an ever-increasing public health burden; the numbers of hip fractures are predicted to be more than 100000 per year by $2020 .^{1-3}$ The latest UK data report an

\author{
Strengths and limitations of this study \\ - Large patient cohort. \\ - Long study period potential confounder. \\ - Potential type two-error despite large sample \\ size. \\ - An observational study hence conclusions are \\ limited.
}

average 30-day mortality of $7.1 \% .^{4}$ One-year mortality rates are reported between $10 \%$ and $30 \%$ with a significantly reduced quality of life among those who survive. ${ }^{3-5}$ Acute hospital and overall length of stay are 16.4 and 21.1 days, respectively, and just over half of patients return to their original residence within 30 days. ${ }^{4}$

Hip fractures mostly, though not exclusively, occur in older people with significant medical and social comorbidity. ${ }^{2}{ }^{3}$ Hip fracture carries a significant socioeconomic burden costing £1-2 billion per year in the $\mathrm{UK}^{6}$

The poor outcomes and wide variations in standards of care led, in April 2010, to the UK Department of Health introducing a financial incentive to English National Health Service (NHS) hospitals. This essentially meant that the 'base' payment made to hospitals for hip fracture care was reduced, but there was additional funding for meeting all of a set of defined process measures: the 'best practice tariff' (BPT). ${ }^{7}$ The best practice tariff criteria were based on national guidance and expert opinion and was intended to drive improvements in processes of care from admission to discharge, where there was evidence of suboptimal practice and where changes in process were felt likely to have the biggest impact. ${ }^{8}$ The criteria are detailed in table 1 and included prompt surgery and the involvement of an orthogeriatrician. The expectation was that patient outcomes would improve as well as reducing length of stay and care costs. ${ }^{9}$ 
Table 1 Best practice tariff criteria

\section{Best practice tariff criteria $^{10}$}

1 Time to surgery with 36 hours from arrival in the A\&E department to the start of anaesthesia (or from time of diagnosis if an admitted patient)

2 Admitted under the joint care of a consultant geriatrician and consultant orthopaedic surgeon

3 Admitted using an assessment protocol agreed by geriatric medicine, orthopaedic surgery and anaesthesia

4 Perioperative assessment by geriatrician in the perioperative period (within 72 hours of admission)

5 Postoperative geriatrician guided multiprofessional rehabilitation team

6 Fracture prevention assessments (falls and bone health)

7 Two AMT scores performed, and all the scores recorded in the NHFD with the first test being carried out prior to surgery and the second post-surgery but within the same spell

Failure of criteria 3 reflects a lack of documentary evidence that the agreed multidisciplinary assessment process was used. AMT, abbreviated mental test.

On the introduction of BPT, the base tariff, payable irrespective of whether the BPT criteria were met, was reduced by $£ 110$. However, should all the BPT criteria be met then an additional payment would be made of $£ 445{ }^{7}$ Subsequent changes to the tariff system have increased this price differential to $£ 1335$. $^{10}$ With a potential uplift of over $£ 1000$ per patient, and compliance monitored via the National Hip Fracture Database, implementation of the hip fracture BPT criteria has been widespread. Current BPT achievement rates are around $63 \%, 100 \%$ compliance is not expected, as some patients will inevitably not be fit for surgery within 36 hours of admission. ${ }^{4}$ Yet increased compliance is often considered to correlate with an increase in quality of care. ${ }^{410-12}$

However, there is limited evidence that increased compliance with BPT has led to improved patient outcomes. There are several published audits demonstrating reduced length of stay following increased compliance of the BPT; however, length of stay is multifactorial and these results were confounded by changes in service provision at reporting hospitals which supported BPT compliance. ${ }^{1112}$ A single study directly assessed the effects of implementing the BPT on mortality, but was unable to demonstrate any survival benefit. ${ }^{13}$ Although there has been an improvement in outcomes that parallels the introduction of BPT, it is not possible to distinguish the possible effects of BPT from more generic improvements in care.

The aim of this study is to determine if the introduction of the BPT has improved outcomes for the hip fracture population and whether achieving the BPT affects an individual's outcome.

\section{METHOD}

This is an observational cohort 'before and after' study. The study was conducted using prospectively collected, anonymised patient data from the Nottingham Hip Fracture Database.

\section{Inclusion and exclusion criteria}

The Nottingham Hip Fracture database ${ }^{14}{ }^{15}$ is a prospectively collected, quality controlled data set based on the European Standardised Audit of Hip Fractures in Europe (SAHFE) process. ${ }^{16}$ Retrospective analysis was performed on two cohorts of patients admitted with a fractured neck of femur to the Queen's Medical Centre. The pre-BPT cohort was from April 2008 until April 2010 and the post-BPT cohort from April 2012 to April 2014. The period between April 2010 and 2011 where BPT was introduced was excluded a priori in order to avoid confounding as the BPT criteria were subsequently changed in 2011 (table 1).

Patients were divided a priori into three groups:

1. Admissions before the implementation of BPT.

2. Admissions after the extended BPT was implemented in 2011 who met the BPT criteria.

3. Admissions after extended BPT implementation but did not achieve the BPT criteria.

Any patient aged under 60 managed non-operatively or who sustained a further hip fracture during the time of the study was excluded.

Mortality and admission data were collected for all patients. Mortality data are provisioned by the Office for National Statistics; the last update of mortality data was June 2015 and all data are censored at that point.

\section{Variables}

Demographic, physiological, operative and admission data were collected for all patients. The Nottingham Hip Fracture Score (NHFS) was prospectively calculated for all patients as part of routine clinical practice. The NHFS is a weighted seven-factor frailty score specific to hip fracture: age; cognitive function on admission (Abbreviated Mental Test (AMT) Score <7); not living at home; sex (male); haemoglobin $<100 \mathrm{~g} / \mathrm{L}$; previous malignancy; $>1$ comorbidity (stroke/transient ischaemic attack; cardiovascular disease; diabetes; previously diagnosed renal disease). It has previously been shown to predict 30-day post hip fracture mortality. The NHFS is a quantitative assessment of the physiological state of the patient and has been shown to be an accurate predictor of 30-day mortality and length of hospital stay within the UK and internationally. ${ }^{15}$ 17-20

\section{Statistical analysis}

The primary outcomes were as follows: differences in mortality in the two cohorts: pre-BPT and post-BPT; and differences in mortality in the achievers and nonachievers in the post-BPT cohort. The primary analysis was performed using 30-day mortality, assessed using $\chi^{2}$ 
tests; complementary analysis was performed using the Cox proportional hazards model.

Secondary analyses were performed using multivariate logistic regression to identify associations between individual BPT criteria and 30-day mortality.

Data were analysed using SPSS statistics programme V.23. Categorical variables are presented as proportions. Ordinal variables are presented as mean or median with IQR as appropriate. Groups were compared with $\chi^{2}$, Student's t-test or Mann-Whitney U test as appropriate. A $\mathrm{p}$ value of $<0.05$ was considered significant. Multivariate logistic regression was performed to identify factors that influenced patient outcomes with 30-day mortality: backward entry, factors with univariate $\mathrm{p}<0.10$ included and $\mathrm{p}<0.05$ as criterion for keeping factors in the model. Formal power analysis was not performed as the sample size is fixed by the nature of the data set.

\section{RESULTS}

A total of 2917 patients were admitted with a hip fracture during the study period. A total of 174 were excluded due to sustaining more than 1 hip fracture; 79 were managed non-operatively; and 123 were aged under 60. This left 2541 patients for analysis of which 1364 were before BPT was introduced and 1177 after BPT. Of the 1177, 314 did not achieve the BPT criteria. Patient characteristics and admission data are summarised in table 2. As previously reported the population characteristics changed over time with more patients admitted from their own home, but an increase in medical complexity demonstrated by an increase in patients with multiple comorbidity, reduction in mobility independence and an increase in average NHFS. ${ }^{3}$

There was no statistically significant difference in 30-day mortality between the pre-BPT cohort and the post-BPT cohort $(113 / 1364(8.3 \%)$ pre-BPT vs $118 / 1177$ $(10.0 \%)$ post-BPT; $\mathrm{p}=0.128)$. Survival analysis showed no difference between the two cohorts either $(\mathrm{p}=0.22)$ (figure 1). NHFS increased from 4.61 (1.47) (mean (SD) in the pre-BPT cohort to 4.74 (1.45) in the post-BPT $(\mathrm{p}=0.026)$.

There was no significant reduction in length of stay 15 days ((IQR 9-21) pre-BPT vs 14 days (IQR 11-22); $\mathrm{p}=0.236$ ) between the two cohorts. The median time from admission to the emergency department to theatre was significantly reduced in the post-BPT cohort (44 hours pre-BPT (IQR 24-44) vs 23 hours post-BPT (IQR 17-30); p<0.005). The proportion of patients being operated on within 36 hours of admission was also significantly higher $(485 / 1364,36 \%$ pre-BPT vs 974/1177, $84 \%$ post-BPT; $\mathrm{p}<0.005)$.

Within the post-BPT cohort, the 30-day mortality was significantly lower in those who achieved BPT (52/863 $(6 \%)$ vs $66 / 314(21 \%)$ in those who did not achieve-BPT; $p<0.005)$. Survival analysis showed a significant long-term survival benefit for those who achieved
BPT (figure 2, $\mathrm{p}<0.005$ ). One-year mortality for those who achieved BPT was $28.6 \%$ (196/863), in comparison to $42.0 \%$ (132/314) for those who did not achieve-BPT $(\mathrm{p}<0.005)$.

Univariate analysis of patient characteristics, their NHFS and the individual NHFS components was performed to identify potential variations between the two groups to explain the difference in mortality rate. Those who did not achieve the BPT criteria had higher NHFS scores, had higher rates of malignancy, were more likely to be man and had lower haemoglobin concentrations (table 1).

Univariate analysis of BPT criteria revealed that time to surgery, orthogeriatrician review, postoperative AMT monitoring, MDT rehabilitation plus falls and bone protection assessment were negatively associated with 30-day mortality (ie, not achieving these criteria was associated with greater 30-day mortality; table 3). Multivariate logistic regression revealed that of the BPT criteria, AMT monitoring and expedited surgery were the only factors that were significantly associated with survival at 30-days and at 1 year (table 4 ).

The commonest cause for failing to meet BPT criteria was a delay in surgery, occurring in approximately a third of cases. All patients were admitted under the joint care of a geriatrician and orthopaedic surgeon. The breakdown of BPT failure and delay to surgery are summarised in tables 5 and 6 .

\section{DISCUSSION}

The introduction of BPT was not associated with a reduction in mortality or length of hospital stay in our hip fracture population. However, at a patient level, failure to achieve BPT was associated with significantly poorer survival. Consistent with NHFD data, delay to surgery is the most common reason not to meet the BPT criteria, despite a significant reduction in the average time from admission to theatre.

The data concerning the impact of BPT are inconsistent. Some studies have reported a temporal association between implementation of BPT and improved outcomes; ${ }^{11}{ }^{12}$ other studies have not demonstrated a change, though this may have been due to inadequate power. ${ }^{13}$ In Wales, which provides data to the National Hip Fracture Database but does not have a BPT mechanism, there have been modest temporal reductions in mortality over a similar time period. As this is an observational study, we are not able to distinguish association and causation. Despite Nottingham having one of the largest hip fracture units in England, combined with a long-standing high-quality clinical database, the analysis is probably underpowered to demonstrate a clinically achievable difference at an organisational level. Of note, mortality in the post-BPT cohort was non-significantly greater than in the pre-BPT cohort. Conversely, the data do support an association at individual level between meeting BPT criteria and outcome. 
Table 2 Patient characteristics and admission data

\begin{tabular}{|c|c|c|c|c|c|c|}
\hline Patient characteristics & Prior to BPT & After BPT & p Value & $\begin{array}{l}\text { Achieved } \\
\text { BPT }\end{array}$ & $\begin{array}{l}\text { Did not } \\
\text { achieve BPT }\end{array}$ & p Value \\
\hline $\mathrm{N}$ & 1364 & 1177 & & 863 & 314 & \\
\hline Median age years (IQR) & $83(77-88)$ & 84 (78-89) & 0.469 & $83(78-89)$ & $82(77-88)$ & $=0.186$ \\
\hline Gender, male:female & $336: 1028$ & $306: 871$ & 0.430 & $200: 663$ & $106: 208$ & $<0.005$ \\
\hline 30-Day mortality N (\%) & $113(8)$ & $118(10)$ & 0.128 & $52(6)$ & $66(21)$ & $<0.005$ \\
\hline Median AMT (IQR) & $8(4-10)$ & $8(4-10)$ & & $8(4-10)$ & $8(4-10)$ & \\
\hline Mean AMT (SD) & $6.71(3.74)$ & $6.73(3.69)$ & 0.826 & $6.74(3.65)$ & $6.68(3.80)$ & 0.85 \\
\hline Median NHFS (IQR) & $4(4-6)$ & $4(4-6)$ & & $4(4-6)$ & $4(4-6)$ & \\
\hline Mean NHFS & 4.61 & 4.72 & 0.026 & 4.69 & 4.88 & $<0.005$ \\
\hline Mean admission $\mathrm{Hb}$ (SD) & $123.8(1.83)$ & $123.3(1.78)$ & 0.435 & $124.0(1.75)$ & $121.0(1.87)$ & 0.03 \\
\hline Median admission Hb (IQR) & $12.5(10.5-14.5)$ & $12.3(10.3-14.3)$ & & & & \\
\hline Malignant fracture (\%) & $163(12)$ & $165(14)$ & 0.121 & $105(12)$ & $60(19)$ & 0.03 \\
\hline Median length of stay (IQR) & $15(9-21)$ & $14(9-19)$ & 0.236 & $18(4-24)$ & $18(3-29)$ & 0.328 \\
\hline $\begin{array}{l}\text { Median time: admission to } \\
\text { theatre (hours) (IQR) }\end{array}$ & $44(23.6-64.4)$ & $23(17-30)$ & $<0.005$ & $21(16-27)$ & $41(27-55)$ & $<0.005$ \\
\hline \multicolumn{7}{|l|}{ Residence } \\
\hline Nursing home (\%) & $151(11)$ & $98(8)$ & 0.001 & $74(8.6)$ & $24(7.6)$ & 0.08 \\
\hline Own home (\%) & $931(68)$ & $869(74)$ & & $625(72)$ & $244(78)$ & \\
\hline Warden aided/residential & 264 (19) & $208(18)$ & & $45(5)$ & $45(14)$ & \\
\hline \multicolumn{7}{|l|}{ home $(\%)$} \\
\hline Hospital inpatient (\%) & $6(0)$ & $0(0)$ & & & & \\
\hline Rehab facility (\%) & $0(0)$ & $2(0)$ & & & & \\
\hline Other (\%) & $9(0)$ & $2(0)$ & & $0(0)$ & $1(0)$ & \\
\hline \multicolumn{7}{|l|}{ Number of comorbidities } \\
\hline$<2(\%)$ & $916(67)$ & $675(57)$ & 0.000 & $508(59)$ & $167(53)$ & 0.08 \\
\hline$\geq 2(\%)$ & $448(33)$ & $502(43)$ & & $355(41)$ & $147(47)$ & \\
\hline Living alone prior to fracture (\%) & $613(45)$ & $496(42)$ & 0.126 & $363(42)$ & $133(42)$ & 0.56 \\
\hline \multicolumn{7}{|l|}{ Walking ability prior to fracture } \\
\hline Independent outdoors (\%) & $668(49)$ & $549(47)$ & 0.002 & $404(47)$ & $145(46)$ & 0.377 \\
\hline Accompanied outdoors (\%) & $231(17)$ & $213(18)$ & & $161(19)$ & $52(17)$ & \\
\hline Independent indoors (\%) & $247(18)$ & $169(14)$ & & $127(15)$ & $42(13)$ & \\
\hline Accompanied indoors (\%) & $76(6)$ & $67(6)$ & & $47(5)$ & $20(6)$ & \\
\hline Unable/transfer only (\%) & $26(2)$ & $25(2)$ & & $13(2)$ & $11(4)$ & \\
\hline Not stated (\%) & $116(9)$ & $154(13)$ & & $110(13)$ & $44(14)$ & \\
\hline
\end{tabular}

Patients in the post-BPT cohort were more frail as demonstrated by a significantly higher NHFS, and individual criteria associated with worse outcome such as male sex, anaemia and malignancy were all more common in the post-BPT cohort. ${ }^{22}$ This may have negated any benefit as a cohort from the introduction of BPT due to the population having a poorer physiological state, and hence worse outcomes despite improvements in perioperative care. Should this failure of BPT to improve outcomes is real and not a type 2 error, then the clinical practice of individual units at the time of its introduction should be considered. The BPT encourages best practice that was already recommended by several national bodies. Expedited surgery and MDT-based rehabilitation were also strongly promoted to prior to its introduction. Hence many of the BPT criteria may have already been implemented within the department and hence introducing BPT would have only improved monitoring of these processes rather than significantly changing the pre-existing model of care.
Failure to achieve BPT at an individual level was associated with poorer outcomes. This suggests that either meeting some or all of the BPT criteria matters or that patients who fail to meet them have pre-existing conditions that are associated with poorer outcomes-or a combination of the two. Those who failed to meet the BPT criteria had a worse NHFS and possessed factors known to adversely affect survival such as being man, anaemia and malignancy. However, the difference in NHFS is relatively small and unlikely to account for all the observed difference in mortality. The two BPT criteria associated with poorer outcomes were delay to surgery and postoperative assessment of cognition.

A significantly higher proportion of men did not achieve BPT (35\% of men did not achieve BPT vs $24 \%$ of women; $\mathrm{p}<0.005)$. Male sex has previously been shown to be an independent predictor of 30-day mortality. ${ }^{14}$ In the post-BPT, cohort men were more likely to have over two comorbidities (158/306 of men had $>2$ comorbidities vs $344 / 871$ of women; $p<0.005)$ and were 
Figure 1 Kaplan-Meier survival analysis comparing long-term survival before and after the introduction of BPT. BPT, best practice tariff.

Figure 2 Kaplan-Meier analysis of long-term survival comparing those who achieved BPT criteria against those who did not. BPT, best practice tariff.
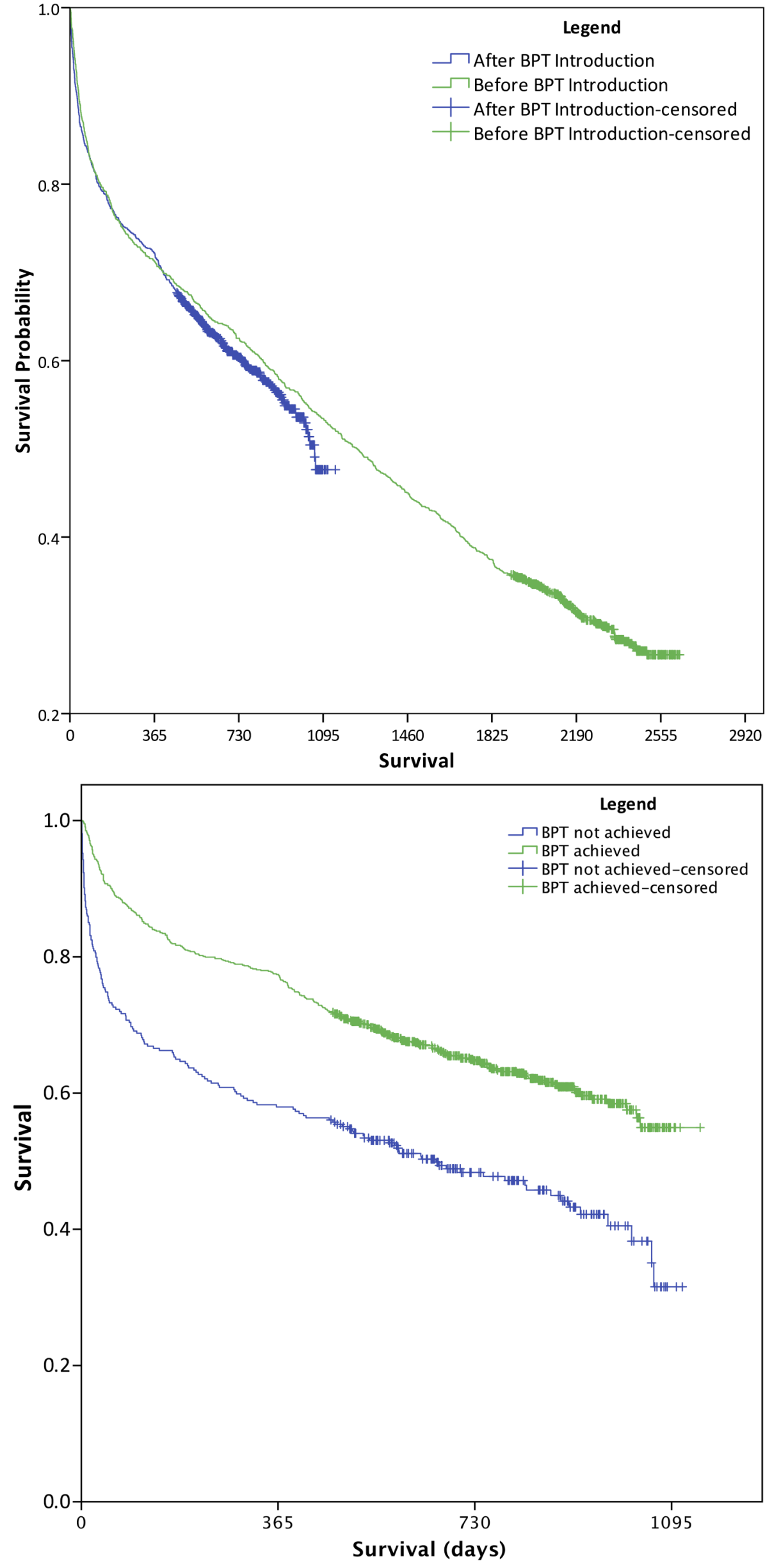
Table 3 Results of univariate analysis of BPT criteria as predictors for 30-day mortality

\begin{tabular}{|c|c|c|c|c|c|c|}
\hline & \multirow[b]{2}{*}{ Survived 30 days } & \multirow[b]{2}{*}{ Did not survive 30 days } & \multirow[b]{2}{*}{ OR } & \multicolumn{2}{|c|}{ OR $95 \% \mathrm{Cl}$} & \multirow[b]{2}{*}{ p Value } \\
\hline & & & & Lower & Upper & \\
\hline Time to surgery & $886 / 1059$ & $85 / 118$ & 0.503 & 0.326 & 0.776 & $<0.005$ \\
\hline MDT admission protocol & $1046 / 1055$ & $115 / 118$ & 0.330 & 0.088 & 1.236 & 0.11 \\
\hline AMT pre-op & $1034 / 1059$ & $116 / 118$ & 1.402 & 0.328 & 5.996 & 0.48 \\
\hline Orthogeriatrician review within 72 hours & $1052 / 1058$ & $110 / 118$ & 0.078 & 0.027 & 0.230 & $<0.005$ \\
\hline AMT post-op & $1013 / 1059$ & $81 / 118$ & 0.099 & 0.061 & 0.162 & $<0.005$ \\
\hline MDT-guided rehabilitation & $1023 / 1057$ & $85 / 114$ & 0.097 & 0.057 & 0.168 & $<0.005$ \\
\hline Falls assessment & $1039 / 1058$ & $103 / 118$ & 0.126 & 0.062 & 0.255 & $<0.005$ \\
\hline Bone protection assessment & $1038 / 1059$ & $102 / 118$ & 0.129 & 0.065 & 0.255 & $<0.005$ \\
\hline
\end{tabular}

Table 4 Results of multivariate logistic regression of BPT criteria as predictors for 30 -day and 1 year mortality

\begin{tabular}{|c|c|c|c|c|c|c|}
\hline & \multicolumn{3}{|c|}{ 30-Day mortality } & \multicolumn{3}{|c|}{1 Year mortality } \\
\hline & B & SE & Sig. & B & SE & Sig. \\
\hline Time to surgery & -0.641 & 0.249 & 0.010 & -0.639 & 0.169 & 0.000 \\
\hline MDT admission protocol & -1.162 & 0.812 & 0.153 & -1.347 & 0.700 & 0.0 .54 \\
\hline AMT pre-op & 2.391 & 0.932 & 0.010 & 2.031 & 0.716 & 0.005 \\
\hline Orthogeriatrician review within 72 hours & -0.988 & 0.777 & 0.204 & 0.393 & 0.768 & 0.609 \\
\hline AMT post-op & -1.740 & 0.393 & 0.000 & -0.642 & 0.355 & 0.071 \\
\hline MDT-guided rehabilitation & -1.234 & 0.446 & 0.006 & -0.819 & 0.400 & 0.041 \\
\hline Falls assessment & -0.474 & 0.939 & 0.614 & 0.003 & 0.831 & 0.997 \\
\hline Bone protection assessment & 0.715 & 0.946 & 0.450 & -1.077 & 0.771 & 0.162 \\
\hline Constant & 0.438 & 1.039 & .673 & 0.895 & 0.954 & 0.348 \\
\hline
\end{tabular}

A p value of $<0.05$ was considered to be statistically significant. Any significant results are highlighted in bold.

Entered on step 1: time to surgery, MDT admission protocol, AMT pre-op, orthogeriatrician review within 72 hours, AMT post-op, MDT-guided rehabilitation, falls assessment and bone protection assessment.

AMT, abbreviated mental test.

more likely to have surgery delayed (66/306 males' time to surgery was $>36$ hours vs $140 / 871$ women; $\mathrm{p}=0.03$ ). The failure of men to meet BPT criteria may be due to their underlying comorbid state delaying surgery while their condition is optimised. However, this association has not been reported previously and may be a chance finding. Replication (or not) of this finding in other units is needed before firm conclusions can be drawn.

A delay to surgery was also the most common reason behind not meeting the BPT, those who did not achieve BPT took twice as long to receive surgery (41 hours vs 21 hours, $\mathrm{p}=0.004$ ). This is despite a significant reduction in the time to surgery after the introduction of BPT (median time to theatre 44 hours pre-BPT vs 23 hours post-BPT, $\mathrm{p}<0.005)$. Expedited surgery is known to be associated with improved survival. ${ }^{23-25}$ However, this may be a reflection of the underlying medical comorbidity requiring optimisation prior to surgery rather than a direct benefit of early surgery itself.

Previous studies have demonstrated no increase in mortality when surgery is delayed up to 4 days when the delay is not due to medical comorbidities. ${ }^{26}$ A delay to surgery of over 36 hours was not associated with an increased NHFS (mean NHFS 4.63, time to theatre $<36$ hours vs mean NHFS 4.72 , time to theatre over
36 hours; $\mathrm{p}=0.08$ ), and the commonest cause for a delay to theatre was a lack of resources rather than the patient being medically unfit (table 5). This, along with an increased NHFS in the post-BPT cohort, may explain why the reduction in time to theatre was not associated with a reduction in mortality.

The association between poor outcome and failure to record an AMT Score postoperatively was unexpected. We regard this result as hypothesis generating but may be a spurious finding. As with delay to surgery, there may be patient factors resulting a failure to document the AMT, or there be an impact on care resulting from this omission, such as missed diagnoses. Patients without a documented AMT may have been clearly confused; pre-existing cognitive impairment and delirium are known risk factors for poorer outcome. ${ }^{27-29}$

Anecdotally, an AMT is omitted when the patient is critically unwell or on an end of life pathway, which might explain the association between 30-day mortality and AMT monitoring. However, the median time to death in those patients who died and missed an AMT was 308 days (IQR 4-708), which perhaps makes this less likely.

The length of stay in hospital did not significantly vary between cohorts, it is known to be multifactorial hence 
Table 5 Breakdown of failure to meet BPT criteria

\begin{tabular}{lr}
\hline Criteria & N (\%) \\
\hline Time to surgery & $100(32)$ \\
AMT post-op & $83(26)$ \\
MDT-based rehabilitation & $63(20)$ \\
Bone protection assessment & $37(12)$ \\
Falls assessment & $34(11)$ \\
AMT recorded pre-op & $27(9)$ \\
Orthogeriatrician review with 72 hours & $14(4)$ \\
MDT admission assessment & $12(4)$ \\
Admission under joint care of surgeon and & $0(0)$ \\
geriatrician & \\
\hline AMT, abbreviated mental test; BPT, best practice tariff. &
\end{tabular}

Table 6 Causes in delay to surgery of over 36 hours

\begin{tabular}{lc}
\hline Cause & N (\%) \\
\hline Lack of resources $^{*}$ & $640(59)$ \\
Medically unfit & $200(18)$ \\
Awaiting investigations & $157(15)$ \\
Deranged coagulation & $57(5)$ \\
Other & $28(3)$ \\
\hline
\end{tabular}

*'Lack of resources' is a broad coding category that can include: a delay due to a caseload with a higher NCEPOD classification, ${ }^{21}$ theatre staff availability and unexpected theatre delays such as prolonged operating time.

the lack of improvement is likely to reflect the availability of medical, nursing and social services to provide support after discharge in a population with low physiological reserve, as well as the postoperative recovery.

The quality and accuracy of the data used in this study is of a high standard; a dedicated audit team prospectively maintain the electronic database which is cross checked for inaccuracy and has previously been shown to have an error rate of $<3 \% .{ }^{25}$ However, despite this, there are limitations to this study. The time over which the data were collected introduces potential confounders, as changes in other aspects of care are likely to have occurred. During this time period, our major trauma network was activated which may have positive and negative effects on aspects of hip fracture care. However, other centres have reported no changes in hip fracture care from becoming a major trauma centre. ${ }^{30}$ The introduction of the BPT is likely to have resulted in a gradual change in practice along with a period of adjustment to the new protocol where the reported results may not reflect actual practice. We attempted to account for this by omitting the year after its introduction from the data.

\section{CONCLUSIONS}

The introduction of the BPT has not led to a demonstrable improvement in outcomes across our hip fracture population. However, during this time period the patients with hip fractures have become significantly more frail with more comorbidities. ${ }^{3}$ There does appear to be a benefit to individual patients associated with achieving BPT. The survival benefit from achieving BPT is potentially due to selection bias, as patients with less comorbidities are less likely to have acute medical problems that would delay surgery or affect their recovery. It may also be that the process of care makes a difference to outcome.

Contributors BO contributed to data and statistical analysis, data interpretation and manuscript preparation. JN contributed to data collation and analysis. CGM involved in the study design, data interpretation and manuscript review. IKM involved in the study design, statistical analysis, data interpretation and manuscript review.

\section{Competing interests None declared.}

Ethics approval The study was registered and accepted with the local audit department and deemed exempt from ethics review in accordance with trust guidelines.

Data sharing statement Data, suitably anonymised and summarised where appropriate, are available on request to the authors.

Open Access This is an Open Access article distributed in accordance with the Creative Commons Attribution Non Commercial (CC BY-NC 4.0) license, which permits others to distribute, remix, adapt, build upon this work noncommercially, and license their derivative works on different terms, provided the original work is properly cited and the use is non-commercial. See: http:// creativecommons.org/licenses/by-nc/4.0/

\section{REFERENCES}

1. Holt G, Smith R, Duncan K, et al. Changes in population demographics and the future incidence of hip fracture. Injury 2009;40:722-6.

2. Cooper C, Campion G, Melton LJ 3rd. Hip fractures in the elderly: a world-wide projection. Osteoporos Int 1992;2:285-9.

3. Baker PN, Salar O, Ollivere BJ, et al. Evolution of the hip fracture population: time to consider the future? A retrospective observational analysis. BMJ Open 2014;4:e004405.

4. Royal College of Physicians. National Hip Fracture Database annual report 2016. London: RCP, 2016.

5. Griffin XL, Parsons N, Achten J, et al. Recovery of health-related quality of life in a United Kingdom hip fracture population. The Warwick Hip Trauma Evaluation-a prospective cohort study. Bone Joint J 2015;97-B:372-82.

6. Lawrence TM, White CT, Wenn R, et al. The current hospital costs of treating hip fractures. Injury 2005;36:88-91.

7. Department of Health. Payment by results guidance for 2010-11. Leeds: Payment by Results team, Department of Health, 2010.

8. British Orthopaedic Association and the British Geriatric Society. The care of patients with fragility fracture. Blue Book Publication by the British Orthopaedic Association, 2007.

9. Royal College of Physicians. Best practice tariff (BPT) for fragility hip fracture care user guide, 2010. http://nhfd.co.uk (accessed 6 Sept 2016).

10. Department of Health. Payment by results guidance for 2013-14. Leeds: Payment by Results Team, Department of Health, 2013.

11. Hawkes D, Baxter J, Bailey C, et al. Improving the care of patients with a hip fracture: a quality improvement report. BMJ Qual Saf 2015;24:532-8.

12. Khan SK, Weusten A, Bonczek S, et al. The best practice tariff helps improve management of neck of femur fractures: a completed audit loop. Brit J Hosp Med (Lond) 2013;74:644-7.

13. Khan SK, Shirley MD, Glennie C, et al. Achieving best practice tariff may not reflect improved survival after hip fracture treatment. Clin Interv Aging 2014;9:2097-102.

14. Maxwell MJ, Moran CG, Moppett IK. Development and validation of a preoperative scoring system to predict 30 day mortality in patients undergoing hip fracture surgery. Br J Anaesth 2008;101:511-17.

15. Moppett IK, Parker M, Griffiths R, et al. Nottingham Hip Fracture Score: longitudinal and multi-assessment. $\mathrm{Br} J$ Anaesth 2012;109:546-50.

16. Parker MJ, Currie CT, Mountain JA, et al. Standardized audit of hip fracture in Europe (SAHFE). Hip Int 1998;8:10-15. 
17. Marufu TC, Mannings A, Moppett IK. Risk scoring models for predicting peri-operative morbidity and mortality in people with fragility hip fractures: qualitative systematic review. Injury 2015;46:2325-34.

18. Karres J, Heesakkers NA, Ultee JM, et al. Predicting 30-day mortality following hip fracture surgery: evaluation of six risk prediction models. Injury 2015;46:371-7.

19. White SM, Moppet IK, Griffiths R, et al. Secondary analysis of prospective observational data from 11085 patients included in the UK Anaesthesia Sprint Audit of Practice (ASAP 2). Anaesthesia 2016;71:506-14.

20. Marufu TC, White SM, Griffiths R, et al. Prediction of 30-day mortality after hip fracture surgery by the Nottingham Hip Fracture Score and the Surgical Outcome Risk Tool. Anaesthesia 2016;71:515-21.

21. The National Confidential Enquiry into Patient Outcome and Death. The NCEPOD classification of intervention, 2004. http://www. ncepod.org.uk/classification.html (accessed 6 Sept 2016)

22. Potter LJ, Doleman B, Moppett IK. A systematic review of pre-operative anaemia and blood transfusion in patients with fractured hips. Anaesthesia 2015;70:483-500.

23. Colais $P$, Di Martino M, Fusco D, et al. The effect of early surgery after hip fracture on 1-year mortality. BMC Geriatr 2015;15:141.
24. Simunovic N, Devereaux PJ, Sprague S, et al. Effect of early surgery after hip fracture on mortality and complications: systematic review and meta-analysis. Can Med Assoc J 2010;182:1609-16

25. Wiles MD, Moran CG, Sahota O, et al. Nottingham Hip Fracture Score as a predictor of one year mortality in patients undergoing surgical repair of fractured neck of femur. $\mathrm{Br} \mathrm{J}$ Anaesth 2011;106:501-4.

26. Moran CG, Wenn RT, Sikand M, et al. Early mortality after hip fracture: is delay before surgery important? J Bone Joint Surg Am 2005;87:483-9.

27. Heruti RJ, Lusky A, Barell V, et al. Cognitive status at admission: does it affect the rehabilitation outcome of elderly patients with hip fracture? Arch Phys Med Rehabil 1999;80:432-6.

28. Van Dortmont LM, Douw CM, van Breukelen AM, et al. Outcome after hemi-arthroplasty for displaced intracapsular femoral neck fracture related to mental state. Injury 2000;31:327-31.

29. Zakriya K, Sieber FE, Christmas C, et al. Brief postoperative delirium in hip fracture patients affects functional outcome at three months. Anesth Analg 2004;98:1798-802.

30. Wong K, Rich J, Yip G, et al. Management of hip fractures preand post-Major Trauma Centre activation. Injury 2015;46: 1975-7. 